\title{
Graphene/hexagonal boron nitride/graphene nanopore for electrical detection of single molecules
}

\author{
Yuhui He, Makusu Tsutsui, Sou Ryuzaki, Kazumichi Yokota, Masateru Taniguchi and Tomoji Kawai
}

Graphene nanopore device, since its proposal, has witnessed tremendous progress toward the goal of single-molecule detection. However, one central challenge of preparing electrodes with nanometer precision on the graphene remains unsolved. Here we show theoretically the feasibility of graphene/hexagonal BN (h-BN)/graphene structure where top graphene layer acts as one electrical contact while the bottom layer as the other. Based on quantum chemistry/nonequilibrium Green's function investigation, we give clear physical pictures why ABC stacking of the above heterogeneous layers results in excellent insulating of the top and bottom graphene electrodes. On the other hand, when the target molecule is inside the nanopore the background conductance through the h-BN dielectric will not keep decreasing even though more layers of h-BN are inside the nanopore. The mechanism is illustrated as that the presence of the molecule will enhance the vertical transmission through the $\mathrm{h}-\mathrm{BN}$ dielectric via quantum interference. We employ a single-level molecule model, and show quantitatively that the discussed effect can be utilized as a powerful signal amplifier for the molecule conductance, thus enhancing the measurability of single molecules by $3-4$ orders.

NPG Asia Materials (2014) 6, e104; doi:10.1038/am.2014.29; published online 6 June 2014

Keywords: ABC stacking; graphene; hexagonal boron nitride; nonequilibrium Green's function; transport properties

\section{INTRODUCTION}

The extreme thinness together with the excellent electrical and mechanical properties of graphene has stimulated great interest in exploring its potential use for single-molecule detection. ${ }^{1-9}$ By fabricating nanogaps or nanopores on the graphene sheet and then propelling single molecules to electrophoretically translocate through the gap/pore, information regarding the target molecules can be recorded and used for characterizing the molecules. This information can be either the longitudinal ionic current blockage due to the transient dwelling of molecule in the gap/pore, ${ }^{2-4,9}$ or the transverse tunneling current through the molecule measured by the electrodes embedded in the pore. ${ }^{1,5,7,8}$ In principle the latter approach is capable of providing knowledge about the molecule electronic structures ${ }^{1,5,8,10}$ and should be quite powerful as a sensor for identifying molecule types. Yet, it is extremely challenging to define electrical contact regions on graphene electrodes and make them aligned with a nanopore. Thus, the realization of tunneling-conductance approach for graphene nanogap/nanopore-based single-molecule detection remains to be reported.

On the other hand, recent successful fabrication of graphene/ hexagonal boron nitride $(\mathrm{G} / \mathrm{h}-\mathrm{BN})$ stacked layers ${ }^{11-13}$ illuminates a potential strategy of insulating the top and bottom graphene sheets by inserting layer(s) of h-BN between them ${ }^{14,15} \mathrm{~h}$-BN sheet, which is known to possess a very large band gap and meanwhile an atomically smooth surface for accommodating the graphene, is expected to be an excellent dielectric for the purpose of blocking the cross-layer conductance. It triggers our idea of designing graphene nanopore device as illustrated in Figure 1a. The top and bottom graphene layers can now be utilized as two separate electrodes for probing the target molecule in the nanopore, as they have been electrically isolated from each other due to the intermediate h-BN layers. The most alluring benefit of the above design is the ultra-fine space resolution that can be provided by the subnanometer-segregated electrodes. Such an atomic-level resolution for electrical measurement may find enormous applications in single-molecule detection. It can be applied, for example, in the field of nanopore-based genome sequencing where the subnanometer distance between adjacent nucleotides on the DNA strands does require such stringent space resolution for discriminating each individual nucleotide.

One crucial concern in this multilayer device architecture is the efficiency. Recent experiments demonstrated that the vertical conductance of bilayer graphene can reach as large as $0.01 \mathrm{~S},{ }^{16}$ while the measured molecule conductance is usually in the range of $10^{-10}-10^{-11} \mathrm{~S} .{ }^{17,18}$ Thereby, a question arises naturally: how many layers of $\mathrm{h}-\mathrm{BN}$ are required for completely impeding the vertical electronic transport through the stacked layers? In order to answer this nontrivial question, a clear physical picture for the insulating mechanism of h-BN dielectric in the graphene/multilayer h-BN/ 


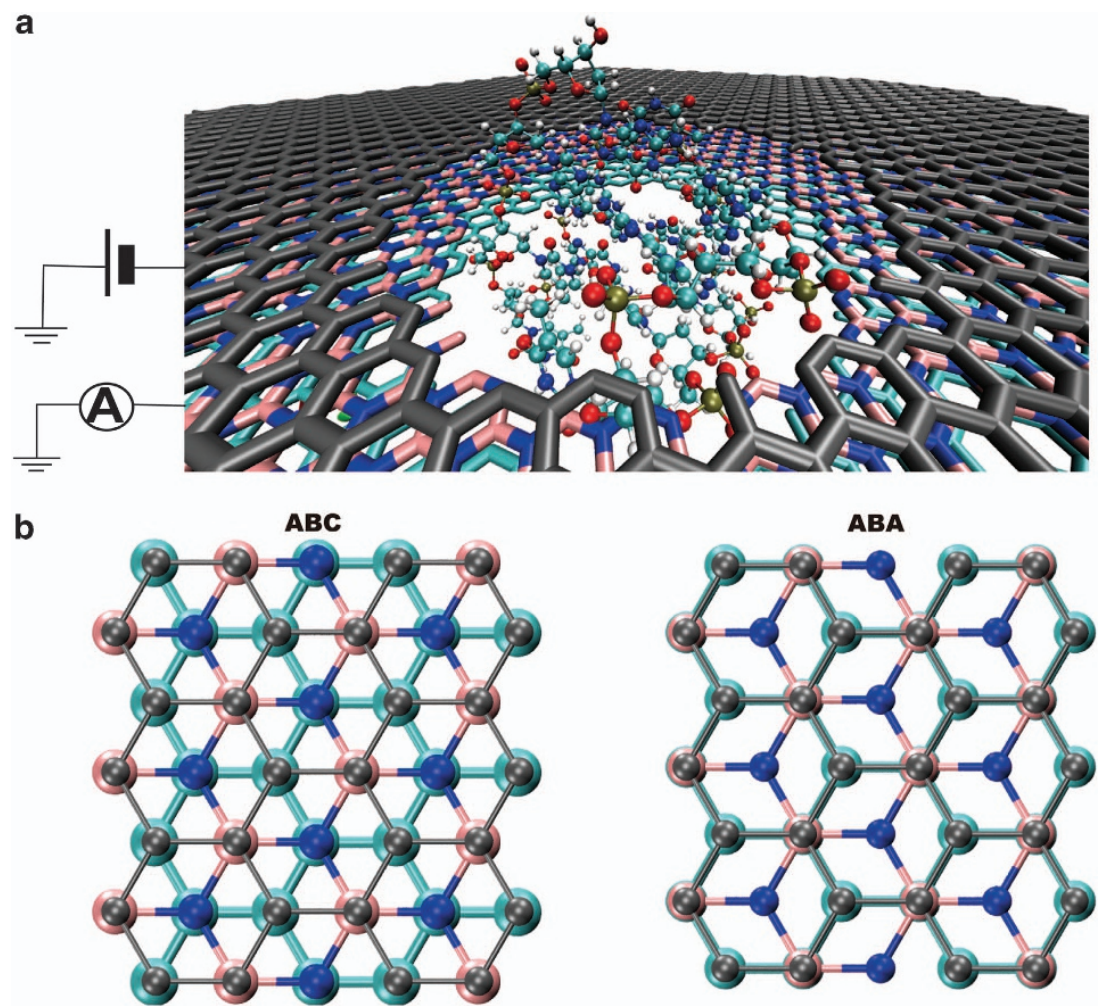

Figure 1 (a) Graphene nanopore with sandwiched h-BN layer for molecule detection. The top and bottom graphene sheets work as two separate electrodes, measuring the vertical electrical conductance through the molecule and the h-BN dielectric. (b) ABC stacking and ABA stacking of G/h-BN/G trilayers: top graphene sheet (gray), intermediate h-BN (pink-blue) and bottom graphene (light blue).

graphene $(\mathrm{G} / x \mathrm{~L}$ h-BN/G) structure and a quantitative examination are urgently demanded. There have been some theoretical studies on the electronic structures of G/h-BN/G trilayer system, ${ }^{19,20}$ and a comparison between tunneling through $\mathrm{G} / \mathrm{h}-\mathrm{BN} / \mathrm{G}$ and h-BN/G/h$\mathrm{BN}$ layers ${ }^{19}$ has been presented. Yet it is rather insufficient as a systematic and comprehensive understanding.

In this work we address the above issue based on quantum chemistry and nonequilibrium Green's function (NEGF) investigation. Starting from the conduction and valence bands of individual graphene and h-BN layers, we demonstrate that inter-layer band coupling is profoundly tuned by the stacking styles. $\mathrm{AB}$ or $\mathrm{BA}$ stacking of G/h-BN leads to entirely different coupling between graphene and h-BN as sketched in Figure 2. The overall consequences are that $A B C$ stacking of $\mathrm{G} / \mathrm{h}-\mathrm{BN} / \mathrm{G}$ will result in the breaking down of conducting path through these heterogeneous layers, while ABA stacking will not. The stacking-sequence-sensitive cross-plane electron transport properties of $\mathrm{G} / \mathrm{h}-\mathrm{BN} / \mathrm{G}$ is far away from a classical view of tunneling-through-barrier effect, and our quantitative calculation shows agreement with the latest experimental reports. ${ }^{14,15}$

Another major finding of this work is the substantial interference between the transmission through the h-BN dielectric and that through the target molecule. We will show that once the target molecule is placed in the $\mathrm{G} / \mathrm{h}-\mathrm{BN} / \mathrm{G}$ nanopore the background conductance through the h-BN dielectric will be profoundly tuned due to the quantum interference with that through the molecule. The result is that the background conductance may not become negligible compared with that caused merely by the molecule even if more layers of h-BN are inside the G/h-BN/G structure. We study the effect quantitatively by using a single-level model for the molecule and show that it can be utilized for attaining enhanced single-molecule measurability.

\section{MATERIALS AND METHODS}

\section{Hamiltonian for G/h-BN/G}

The most stable configuration of G/h-BN stacking has been shown as that one of the two inequivalent carbon atoms in a graphene primitive cell is on top of a boron atom while the other one sits above the center of the boron nitride ring. ${ }^{21,22}$ The associated $A B A$ and $A B C$ stacking models of $\mathrm{G} / \mathrm{h}-\mathrm{BN} / \mathrm{G}$ trilayers are depicted in Figure 1b. In the vicinity of the highly symmetric $\boldsymbol{K}$ and $\boldsymbol{K}^{\prime}$ points in the $\boldsymbol{k}$-space, the Hamiltonian of ABC-stacked G/h-BN/G trilayer system can be written as follows ${ }^{23}$ (detailed derivation is provided in the Supplementary Material):

$$
H_{\mathrm{ABC}}=\left[\begin{array}{cccccc}
\varepsilon_{C}+\frac{U}{2} & v_{f} k e^{i \theta_{\vec{k}}} & 0 & 0 & 0 & 0 \\
& \varepsilon_{C}+\frac{U}{2} & \gamma_{1} & 0 & 0 & 0 \\
& & \varepsilon_{B} & v_{B N} k e^{i \theta_{\vec{k}}} & 0 & 0 \\
& & & \varepsilon_{N} & \gamma_{1^{\prime}} & 0 \\
& \text { h.c. } & & & \varepsilon_{C}-\frac{U}{2} & v_{f} k e^{i \theta_{\vec{k}}} \\
& & & & & \varepsilon_{C}-\frac{U}{2}
\end{array}\right]
$$

In the above equation, $\varepsilon_{B}, \varepsilon_{N}$ and $\varepsilon_{C}$ are the $2 p_{z}$ on-site energies of boron, nitrogen and carbon sublattices, $v_{f}$ and $v_{B N}$ are related with the hopping parameters between the nearest neighboring atoms in the graphene and h-BN sheets respectively, while $\gamma_{1\left(^{\prime}\right)}$ denotes the inter-layer coupling between vertically aligned carbon and boron/nitrogen atoms; $\boldsymbol{k}$ is the in-plane wavevector, and $\theta_{\boldsymbol{k}}$ is its angle; $U$ is the applied vertical voltage. The values of the above parameters have been obtained by fitting with $a b$ initio calculations ${ }^{23}$ and are listed in the end of this section. On the other hand, the Hamiltonian 

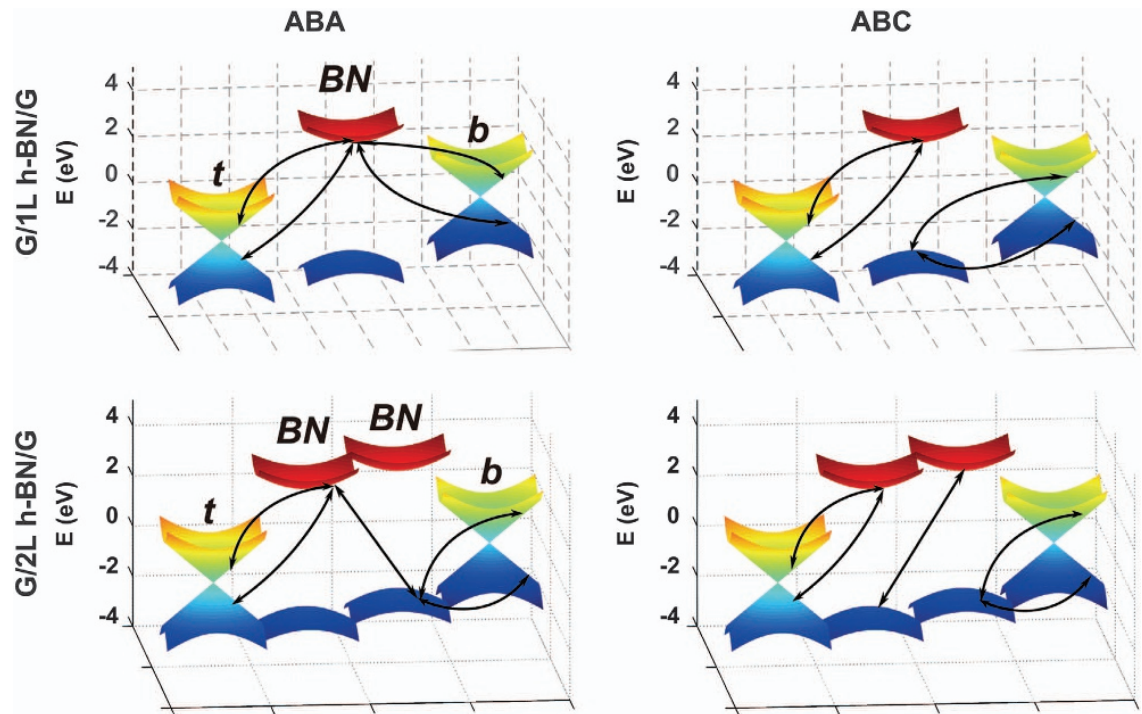

Figure 2 The cross-layer electrical conducting mechanism near the $\boldsymbol{K}$ points of graphene and h-BN energy bands: for the upper half there is $1 \mathrm{~h}$-BN layer inside as G/1L h-BN/G, while for the lower half there are 2 layers as G/2L h-BN/G; for the left half the graphene and h-BN are ABA-stacked, while for the right half they are $A B C$-stacked. It demonstrates that for ABA stacking near the Dirac points there exists always a conducting path from the top graphene sheet to the bottom one, whereas for ABC stacking such a path does not exist.

for ABA-stacked G/h-BN/G trilayer system is as follows ${ }^{23}$ :

$$
H_{\mathrm{ABA}}=\left[\begin{array}{cccccc}
\varepsilon_{C}+\frac{U}{2} & v_{f} k e^{i \theta_{\vec{k}}} & 0 & 0 & 0 & 0 \\
& \varepsilon_{C}+\frac{U}{2} & \gamma_{1} & 0 & 0 & 0 \\
& & \varepsilon_{B} & v_{B N} k e^{i \theta_{\vec{k}}} & 0 & \gamma_{1} \\
& & & \varepsilon_{N} & 0 & 0 \\
& \text { h.c. } & & & \varepsilon_{C}-\frac{U}{2} & v_{f} k e^{i \theta_{\vec{k}}} \\
& & & & & \varepsilon_{C}-\frac{U}{2}
\end{array}\right]
$$

Yet we remind the above is a conventional treatment of constructing Hamiltonian because it relies on a basis as $2 p_{z}$ orbitals of the two inequivalent sublattices on each layer with Bloch factors. ${ }^{23}$ In this work we deliberately choose to discuss in the framework of conduction and valence $\pi$-bands of individual graphene and h-BN layers. Mathematically, it means we transfer into another representation where $|\Psi\rangle=\left\{\left|\Psi_{\vec{k}}^{t, c}\right\rangle\left|\Psi_{\vec{k}}^{t, v}\right\rangle\left|\Psi_{\vec{k}}^{B N, c}\right\rangle\left|\Psi_{\vec{k}}^{B N, v}\right\rangle\left|\Psi_{\vec{k}}^{b, c}\right\rangle\left|\Psi_{\vec{k}}^{b, v}\right\rangle\right\}$. The superscripts ${ }^{t}$ and ${ }^{b}$ denote the top and bottom graphene layers, while ${ }^{c}$ and ${ }^{v}$ indicate conduction and valence bands. In this manner, the Hamiltonian for ABC-stacked G/h-BN/G trilayers becomes (detailed derivation is shown in the Supplementary Material):

$$
H_{\mathrm{ABC}}=\left[\begin{array}{ccc}
H_{t} & V_{t, B N} & 0 \\
V_{B N, t} & H_{B N} & V_{B N, b} \\
0 & V_{b, B N} & H_{b}
\end{array}\right]
$$

where $H_{t(b)}$ and $H_{B N}$ are intra-layer Hamiltonian for top (bottom) graphene sheets and the sandwiched BN layer, while $V_{t(b), B N}$ is inter-layer coupling between top (bottom) graphene and the BN. The Hamiltonian for the individual top/bottom graphene layer becomes diagonalized as $H_{t, c(v)}=$ $\varepsilon_{C} \pm v_{f} k+U / 2$ and $H_{b, c(v)}=\varepsilon_{C} \pm v_{f} k-U / 2$,

$$
H_{t(b)}=\left[\begin{array}{cc}
H_{t(b), c} & 0 \\
0 & H_{t(b), v}
\end{array}\right]
$$

while that for the central h-BN is $H_{B N, c(v)}=\bar{\varepsilon}_{B N} \pm \sqrt{\Delta \varepsilon_{B N}^{2}+v_{B N}^{2} k^{2}}$, where $\bar{\varepsilon}_{B N}=\left(\varepsilon_{B}+\varepsilon_{N}\right) / 2$ and $\Delta \varepsilon_{B N}-\left(\varepsilon_{B}-\varepsilon_{N}\right) / 2$.

$$
H_{B N}=\left[\begin{array}{cc}
H_{B N, c} & 0 \\
0 & H_{B N, v}
\end{array}\right]
$$

These bands are visualized by the curved surfaces in Figure 2. Moreover, the inter-layer coupling matrices now are as follows:

$$
\begin{aligned}
V_{t, B N} & =\frac{v_{B N} k e^{i \theta_{\vec{k}}} \gamma_{1}}{\sqrt{2}}\left[\begin{array}{cc}
-\frac{1}{a_{1}} & -\frac{1}{a_{2}} \\
\frac{1}{a_{1}} & \frac{1}{a_{2}}
\end{array}\right] \\
V_{b, B N} & =\frac{e^{i \theta_{\vec{k}} \gamma_{1^{\prime}}}}{\sqrt{2} \Delta \varepsilon_{B N}}\left[\begin{array}{cc}
\frac{a_{1}^{2}-2 v_{B_{N}}^{2} k^{2}}{2 a_{1}} & \frac{a_{2}^{2}-2 v_{v_{N}}^{2} k^{2}}{2 a_{N}} \\
\frac{a_{1}^{2}-2 v_{B N}^{2} k^{2}}{2 a_{1}} & \frac{a_{2}^{2}-2 v_{B N}^{2} k^{2}}{2 a_{2}}
\end{array}\right]
\end{aligned}
$$

In the above expression $\vec{k}=\left(k, \theta_{\vec{k}}\right)$ is the in-plane wave-vector expressed in the polar coordinate, and $a_{1(2)}=\sqrt{2 \Delta \varepsilon_{B N}^{2}+2 v_{B N}^{2} k^{2} \mp \sqrt{\Delta \varepsilon_{B N}^{2}+v_{B N}^{2} k^{2}}}$.

Now here comes the central finding for the vertical conductance of heterogeneous ABC-stacked G/h-BN layers: under small doping concentration, that is, $k \rightarrow 0$, the coupling matrices between the graphene and the neighboring h-BN become as follows:

$$
\begin{aligned}
& \begin{array}{cc}
\left\langle\Psi_{\vec{k}}^{t, c}\right| & \left|\Psi_{\vec{k}}^{B N, c}\right\rangle \\
\left\langle\Psi_{\vec{k}}^{t, v}\right| & \left|\Psi_{\vec{k}}^{B N, v}\right\rangle
\end{array} \quad \times \frac{\gamma_{1} e^{i \theta_{\vec{k}}}}{\sqrt{2}}
\end{aligned}
$$

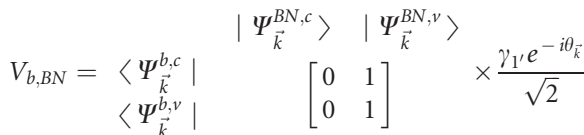

The above expressions have fairly clear physical interpretations as shown in the upper-right of Figure 2: the $\mathrm{AB}$ stacking of top graphene and h-BN will result in hopping of both electrons and holes in the graphene sheet to the conduction band of h-BN, while BC stacking of h-BN and bottom graphene, which is in fact $A B$ stacking of h-BN and bottom graphene, will lead to hopping of graphene carriers to the valence band of $\mathrm{h}-\mathrm{BN}$. These diagrams are depicted by the corresponding arrows in Figure 2. It predicts that in the absence of effective coupling between h-BN conduction and valence bands, there will be no conducting path from top graphene sheet to the bottom one. Usually such inter-valley coupling is offered by impurity scattering or phonon scattering in two-dimensional hexagonal materials, ${ }^{24,25}$ and in h-BN it would be very small due to the very large band gap. On the other hand, given the symmetry of the top and bottom graphene layers with respect to the central h-BN one in the ABA stacking, there will always be an effective conducting path through the trilayers as shown in the upper-left of Figure 2. Thus we reveal an absolutely 
different insulating mechanism compared with the classical tunnelingthrough-barrier explanation: The conducting path will be switched on or off depending on the $\mathrm{G} / \mathrm{h}-\mathrm{BN} / \mathrm{G}$ stacking sequence. We remind that previous density functional calculation showed similar results that the tunneling transport through stacks of graphene and h-BN depends sensitively on the stacking order. ${ }^{19}$ Here we present a clear physical picture why such dependence occurs.

In the case there are more than one layer of h-BN fabricated between the top and bottom graphene sheets $\left(\mathrm{G} / x \mathrm{~L}\right.$ h-BN/G), ${ }^{15}$ the Hamiltonian of $\mathrm{AB}$-stacked $\mathrm{h}-\mathrm{BN} / \mathrm{h}-\mathrm{BN}$ is written as follows in the sublattice Bloch wave representation:

$$
H_{B N}=\left[\begin{array}{ll}
H_{1} & V_{12} \\
V_{21} & H_{2}
\end{array}\right]
$$

With the intra-layer BN Hamiltonian

$$
H_{1(2)}=\left[\begin{array}{cc}
\varepsilon_{B}+U_{1(2)} & v_{B N} k e^{i \theta_{\vec{k}}} \\
v_{B N} k e^{-i \theta_{\vec{k}}} & \varepsilon_{N}+U_{1(2)}
\end{array}\right]
$$

and the inter-layer counterpart

$$
V_{12}=\left[\begin{array}{cc}
0 & 0 \\
\gamma_{2} & 0
\end{array}\right]
$$

In the above expression, the subscripts ${ }_{1}$ and ${ }_{2}$ denote the upper and lower h-BN sheets respectively, and $\gamma_{2}$ is the parameter characterizing coupling strength between the two BN sheets. ${ }^{15}$ After transforming to the representation of eigen-states of individual sheets $\left\{\left|\Psi_{\vec{k}}^{1, c}\right\rangle\left|\Psi_{\vec{k}}^{1, v}\right\rangle\left|\Psi_{\vec{k}}^{2, c}\right\rangle\left|\Psi_{\vec{k}}^{2, v}\right\rangle\right\}$, the intra-layer BN Hamiltonian $H_{1(2)}$ gets diagonalized as similar to that shown in Equation (5), while the inter-layer coupling $V_{1(2)}$ between the $\mathrm{AB}$-stacked two h-BN layers now becomes (see derivation detail in the Supplementary Material):

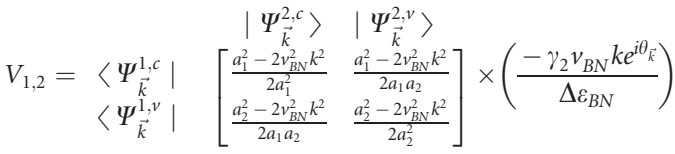

Again, $\left|\Psi_{\vec{k}}^{1(2), c}\right\rangle$ and $\left|\Psi_{\vec{k}}^{1(2), v}\right\rangle$ denotes the conduction and valence states of the individual BN sheets. Near the Dirac points, it becomes

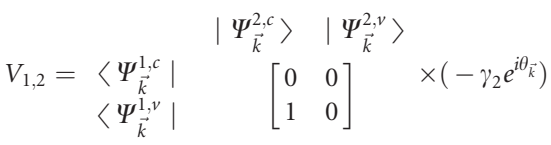

As depicted in the lower-right of Figure 2, upon AB stacking of h-BN/h-BN, only the coupling between valence band of the upper h-BN and conduction band of the lower h-BN survives. The figure further demonstrates the overall consequences as that $\mathrm{ABC}$ stacking of $\mathrm{G} / 2 \mathrm{~L}$ h-BN/G will result in the breaking down of conducting path through these heterogeneous layers, while ABA stacking of the quadri-layers will not. More generally, there are three styles of inter-layer band coupling depicted as AB-stacked G/h-BN, BA stacked G/h-BN and $\mathrm{AB}$-stacked $\mathrm{h}-\mathrm{BN} / \mathrm{h}-\mathrm{BN}$, which have been clearly marked by the arrows in Figure 2 (here we remind that $\mathrm{BC}$ staking is the same as the $\mathrm{AB}$ ), and arbitrary number of sheets of $\mathrm{ABA}$ or $\mathrm{ABC}$ stacking can always be evaluated as a combination of the above three types in series. By utilizing these diagrams, we perform similar plotting of the conducting paths for increasing number of h-BN layers. As an example, the diagrams for G/3L h-BN/G structure stacked in $\mathrm{ABC}$ and $\mathrm{ABA}$ models are presented in Supplementary Figure $\mathrm{S} 2$ of the Supplementary Material. Then, the conclusion is as readily seen: the ABABAB stacking of $\mathrm{G} / x \mathrm{~L}$ h-BN/G will always preserve a conducting path for the crosslayer carriers, whereas $\mathrm{ABCABC}$ will never do so.

The cross-layer electrical current is then expressed by the transmission function $T(E, \vec{k})$ according to the Landauer formula

$$
I=\int \mathrm{d} E \sum_{\vec{k}} T(E, \vec{k})\left[f\left(E-\mu_{t}\right)-f\left(E-\mu_{b}\right)\right]
$$

where $f(E)$ is the Fermi distribution function, and $\mu_{t(b)}$ is the chemical potential of top/bottom graphene electrodes. $T(E, \vec{k})$ is evaluated via the nonequilibrium Green's function formalism:

$$
T(E, \vec{k})=\operatorname{Tr}\left(\Gamma_{t} G(E, \vec{k}) \Gamma_{b} G^{+}(E, \vec{k})\right)
$$

In the above expression, $\Gamma_{t(b)}=i\left(\Sigma_{t(b)}-\Sigma_{t(b)}^{+}\right)$and $G$ is the full Green's function of the $\mathrm{BN}$ layers (the superscript ${ }^{+}$means Hermitian conjugate):

$$
G(E, \vec{k})=\frac{1}{E-H_{B N}(\vec{k})-\Sigma_{t}(E, \vec{k})-\Sigma_{b}(E, \vec{k})}
$$

The self-energies of top and bottom graphene layers for ABC stacking are calculated via $\Sigma_{t(b)}(E, \vec{k})=V_{t(b), B N}^{+} g_{t(b)} V_{t(b), B N}$ and are presented as follows:

$$
\begin{aligned}
& \Sigma_{t}(E, \vec{k})=\frac{\left|\gamma_{1}\right|^{2}}{2} v_{B N}^{2} k^{2}\left(g_{t, c}(E, \vec{k})+g_{t, v}(E, \vec{k})\right)\left[\begin{array}{cc}
\frac{1}{a_{1}^{2}} & \frac{1}{a_{1} a_{2}} \\
\frac{1}{a_{1} a_{2}} & \frac{1}{a_{2}^{2}}
\end{array}\right] \\
& \Sigma_{b}(E, \vec{k})=\frac{\left|\gamma_{1^{\prime}}\right|^{2}}{2}\left(g_{b, c}(E, \vec{k})+g_{b, v}(E, \vec{k})\right)\left[\begin{array}{cc}
1-\frac{v_{B N}^{2} k^{2}}{a_{1}^{2}} & \frac{1}{a_{1} a_{2}} \\
\frac{1}{a_{1} a_{2}} & 1-\frac{v_{B N}^{2} k^{2}}{a_{2}^{2}}
\end{array}\right]
\end{aligned}
$$

In the above, $g_{t(\mathrm{~b}), c}$ and $g_{t(\mathrm{~b}), v}$ are Green's functions of conduction electrons and valence holes in the top (bottom) graphene layers:

$$
g_{t, c(v)}=\frac{1}{E-\left(U_{t}+\varepsilon_{C} \pm v_{f} k\right)-\Sigma_{p h}-\Sigma_{i m}}
$$

where $\Sigma_{p h}$ and $\Sigma_{i m}$ are self-energies due to electron-phonon ${ }^{26,27}$ and impurity scattering. ${ }^{24,25}$ The expressions and parameters will be listed in the end of this section.

\section{Molecule conductance}

With the under-interrogation molecule dwelling in the nanopore, the basis is extended to include the molecule wavefunction $\left|\Phi_{M}\right\rangle=$ $\left\{\left|\phi_{1}\right\rangle\left|\phi_{2}\right\rangle \ldots\left|\phi_{i}\right\rangle \ldots\right\}$, which is a linear combination of atomic orbitals on the molecule. The system is now divided into three parts: top and bottom graphene electrodes, and the extended molecule including the sandwiched $\mathrm{h}$-BN layers and the target molecule. Hamiltonian for the extended molecule now becomes:

$$
H_{\text {ext }}=\begin{array}{cc}
\left|\Psi_{\vec{k}}^{B N}\right\rangle & \left|\Phi_{M}\right\rangle \\
& \left\langle\Phi_{M}^{B N}\right|
\end{array} \quad\left[\begin{array}{cc}
H_{B N} & V_{B N, M} \\
V_{M, B N} & H_{M}
\end{array}\right]
$$

and the element of coupling between top (bottom) graphene electrode and the molecule $V_{t(b), M}$ is evaluated as follows:

$$
V_{t, \alpha ; M, i}=\left\langle\Psi_{\vec{k}}^{t, \alpha}|\hat{H}| \phi_{i}\right\rangle=\frac{1}{\sqrt{N}} \sum_{\vec{R}_{t, \alpha}^{\prime}} \phi_{\pi}^{*}\left(\vec{r}-\vec{R}_{t, \alpha}^{\prime}\right) H(\vec{r}) \phi_{i}(\vec{r})
$$

Here $\vec{R}_{t, \alpha}^{\prime}$ are those atoms on the $\alpha$-sublattice of top graphene layer that have overlaps with the target molecule, and $N$ is the number of primitive cells on the graphene. We denote the above equation as $V_{t, \alpha ; M, i}=V_{t, \alpha ; i} l \sqrt{ } N$. For the evaluation of real molecules a standard $a b$ initio-NEGF calculation will be implemented, while at current stage in order to clearly figure out the physical mechanism, we employ a single-level model for the target molecule. We further assume the molecule energy level is $E_{M}=0 \mathrm{eV}$, and its couplings with edge atoms on the two sublattices of both top and bottom graphene electrodes are kept at $V_{t(b), \alpha ; M}=V_{t(b), \beta ; M}=0.4 / \sqrt{ } N$ e $\mathrm{V}^{2}$ a bias voltage $U=0.2 \mathrm{~V}$ is applied and the electron doping concentrations of the graphene electrodes are $N_{c}=10^{10} \mathrm{~cm}^{-2}$.

Parameters used in the calculation: $\varepsilon_{B}=3.34 \mathrm{eV}, \varepsilon_{N}=-1.40 \mathrm{eV}, v_{f}=10^{6}$ $\mathrm{ms}^{-1}, v_{B N}=1.057 \times 10^{6} \mathrm{~ms}^{-1}$ and $\gamma_{1}=0.43 \mathrm{eV}$, while the graphene Dirac point $\varepsilon_{C}$ is set at $0 \mathrm{eV}$. The expressions for self-energies in equation (20) are

$$
\begin{aligned}
\operatorname{Im}\left[\Sigma_{p h}\right]= & \frac{\sqrt{3}}{16} a^{2} \alpha_{g}^{2} g^{2}\left|E-\operatorname{sgn}\left(E-E_{f}\right) \hbar \omega_{\mathrm{ph}}-E_{D}\right| \theta\left(\left|E-E_{f}\right|-\hbar \omega_{\mathrm{ph}}\right) \\
& \theta\left(w-\left|E-E_{D}\right|\right) \\
\operatorname{Im}\left[\Sigma_{\mathrm{im}}\right]= & N_{\mathrm{im}} \pi^{2} \alpha^{2} \frac{E}{\left(E / v_{f}+q_{T F}\right)^{2}}
\end{aligned}
$$

In the above expression, $\operatorname{Im}[\Sigma]$ is the imaginary part of the self-energy, $a$ is the lattice parameter of graphene sheet, $\alpha_{g}=2.53$ is the effective fine structure 

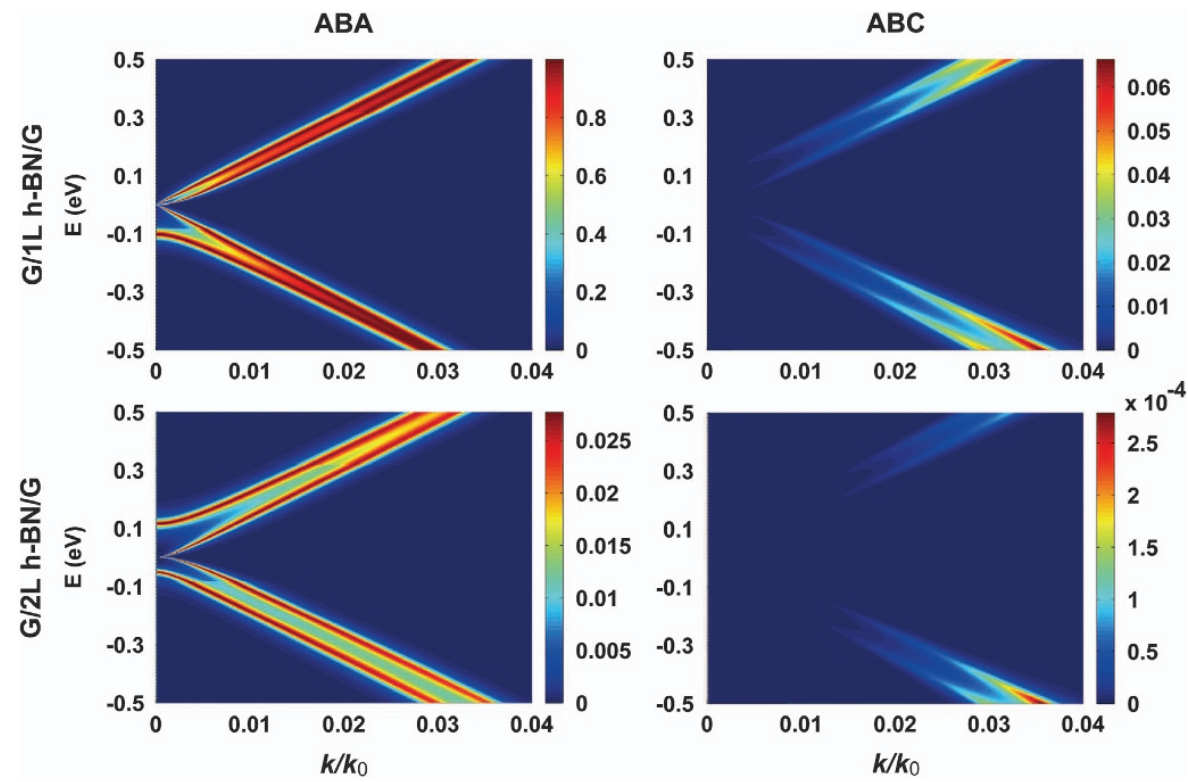

Figure 3 The calculated transmission spectrum $T(E, k)$ from top graphene layer to the bottom one. Results are arranged in the same order as in Figure 2 . Here the graphene layers are assumed to be $\mathrm{N}$-doped with doping concentration $N_{c}=10^{12} \mathrm{~cm}^{-2}$, and thus the corresponding $E_{f}=E_{D}+0.117 \mathrm{eV}$. The systems are unbiased $U=0$ while $E=0 \mathrm{eV}$ is set at the Dirac point of graphene.

constant of graphene, $g=3.5 \times 10^{-2} \mathrm{Ry}$ is the average electron-phonon matrix element in Rydberg unit, $\hbar \omega_{\mathrm{ph}}=0.2 \mathrm{eV}$ is the optical phonon energy, $E_{f}$ is the Fermi level of the doped graphene sheet, $w$ is the width of graphene $\pi$-band, $\alpha=0.75$ and $q_{T F}=4 \alpha \sqrt{\pi} N_{\text {im }}$ is the wave-number at the Fermi level of graphene sheet.

\section{RESULTS}

Cross-layer conductance

The quantitative calculation results by using the Landauer formula and nonequilibrium Green's function technique (calculation details are given in the Supplementary Material) are presented in Figure 3. Here the graphene layers are assumed to be n-doped with concentration $N_{c}=10^{12} \mathrm{~cm}^{-2}, 28$ which corresponds to Fermi energy $E_{f}=E_{D}+0.12 \mathrm{eV}\left(E_{D}\right.$ is the energy of graphene Dirac point). The zero-bias transmission spectrums $T(E, k)$ are demonstrated in the upper-row for $\mathrm{ABA}$ and $\mathrm{ABC}$-stacked $\mathrm{G} / \mathrm{h}-\mathrm{BN} / \mathrm{G}$ trilayers respectively $\left(E=0\right.$ is set at the graphene Dirac point $\left.E_{D}\right)$. In the interested energy range, there are two bright lines starting from the same point $(E, k)=(0,0)$ and becoming divergent from each other when $k$ grows larger. These are corresponding to the tunneling of graphene electrons and holes, since they manifest exactly the energy dispersion relation of graphene sheets. Besides, there exist two much weaker transmission lines starting from the point $(E, k)=(-1.40,0)$ and $(E, k)=(3.34,0)$ as shown in Supplementary Figure S2 of Supplementary Material where a larger energy range is exhibited. They are the transmission caused by h-BN electron and hole transport. By comparing the ABA and $\mathrm{ABC}$ stacking cases, we conclude that $\mathrm{ABC}$ stacking does block the tunneling of carriers from top to bottom graphene layers. Moreover, if there are two h-BN layers sandwiched and the layers are ABC-stacked, the transmission will be further reduced by two orders as shown in the lower row of Figure 3. In such a quadri-layer system, the tunneling by the graphene electrons and holes is almost eliminated when $|E|<0.1 \mathrm{eV}$ and $|k| \ll k_{0}$. The physical implication is that under low doping concentration $\left(\left|k_{F}\right| \ll k_{0}\right)$ and small bias $(U<0.1 \mathrm{~V})$ the cross-layer conductance in ABC-stacked G/2L h-BN/G system can be neglected. This is further shown in Table 1 where the
Table 1 Cross-layer conductance under $U=200 \mathrm{mV}\left(\mu \mathrm{S} \mu \mathrm{m}^{-2}\right)$

\begin{tabular}{lcccc}
\hline & $G / G / G$ & $G / h-B N / G$ & $G / 2 L h-B N / G$ & $G / 3 L h-B N / G$ \\
\hline ABA stacking & $7.8 \times 10^{2}$ & $4.5 \times 10^{2}$ & 99 & 7.6 \\
ABC stacking & $4.1 \times 10^{2}$ & 12 & $1.5 \times 10^{-2}$ & $1.6 \times 10^{-6}$ \\
Experiments $^{14}$ & & 1000 & 50 & 1 \\
\hline
\end{tabular}

calculated vertical conductance per-square micrometer of ABA- and ABC-stacked G/h-BN/G layers is listed $\left(N_{c}=10^{10} \mathrm{~cm}^{-2}\right.$ and $\left.E_{f}=E_{D}+10^{-3} \mathrm{eV}^{15}\right)$. As a reference, that of trilayer graphene $\mathrm{G} / \mathrm{G} / \mathrm{G}$ is also presented. It indicates that the vertical conductance of $\mathrm{G} / \mathrm{h}-\mathrm{BN} / \mathrm{G}$ trilayers will drop about two orders if piled in the $\mathrm{ABC}$ style. Furthermore, by introducing one more layer of h-BN the conductance will decrease three to four orders with the $\mathrm{ABC}$ stacking structure while two orders in ABA case. We find that for the ABA stacking case, both the calculated conductance and its variance trend with the number of h-BN sheets, show agreement with the experimental observation. ${ }^{15}$

There are two potential explanations concerning the experimentally fabricated G/h-BN/G. One is that these heterogeneous layers are ABAstacked due to energy favorableness, and the other is they are piled up in a lattice constant mismatched manner. The first possibility is based on the fact that the total energy of ABA stacking is smaller than that of $\mathrm{ABC}$ stacking, and thus the former should possess a higher preference during the fabrication than the latter. Here the physical mechanism is that ABA-stacked G/h-BN/G trilayers can be viewed as the sum of nitrogen-centered (N-centered) G/h-BN and N-centered $\mathrm{h}-\mathrm{BN} / \mathrm{G}$ again, while $\mathrm{ABC}$-stacked ones are the add-up of N-centered G/h-BN and then boron-centered (B-centered) h-BN/G. For G/h-BN bilayer stacking the most energetically favorable structure is the $\mathrm{N}$-centered piling as indicated by density functional calculations. ${ }^{21}$ Therefore, we conclude ABA stacking should be more preferred than $\mathrm{ABC}$ case. Quantitatively, the difference is $\sim 3.9 \mathrm{meV}$ per atom, ${ }^{22}$ which suggests a gap of $306 \mathrm{meV} \mathrm{nm}^{-2}$ between ABA- and ABC stacking. The other plausible explanation is that the $\mathrm{G} / \mathrm{h}-\mathrm{BN} / \mathrm{G}$ layers 
do not match each other's lattice constant. This is clearly demonstrated by Moire patterns in the latest experiments related to G/h-BN layers. ${ }^{13,29}$ In this case the intermediate h-BN layers serve as pure tunneling barriers while the quantum interference effect is neglected. ${ }^{15}$ Yet for the purpose of nanopore-based single-molecule detection, we suggest $\mathrm{ABC}$ stacking since Table 1 indicates that the vertical conductance of G/3L h-BN/G will be as small as $2 \mathrm{pS} \mu \mathrm{m}^{-2}$. We put the discussion of the physical mechanism and strategies for experimental fabrication in the 'Commensurate and incommensurate stacking' section of Supplementary Material.

\section{Molecule conductance}

The above analysis demonstrates that the ordered stacking of h-BN dielectric layers will lead to quantum interference effect on the crosslayer conductance. Will it also modulate the molecule conductance profoundly, which cannot be depicted by the turbostratic stacking of h-BN dielectric? In this subsection we are going to show our investigation on this topic.

Figure 4 plots the transmission spectrums in logarithm scale $\log _{10} T$ when the target molecule dwells in an ABC-stacked nanopore, while the number of sandwiched h-BN sheets $N_{B N}$ varies from 1-4. By comparing Figures $4 \mathrm{a}$ and $\mathrm{b}$ we find that with only one layer of h-BN inside, the transmission caused by the graphene electron and hole tunneling is observed while that caused by the molecule near $E=0 \mathrm{eV}$ cannot be found. The absence of molecule signal is just as expected because one layer of h-BN dielectric is too weak to insulate the electron tunneling between top and bottom graphene electrodes. As a result, the molecule conductance is completely immersed in the background conductance. Then, if $N_{B N}$ increases from 2 to 3, the transmission line caused by the molecule becomes evident, as the background conductance turns darker. However, it is intriguing to see that the background transmission no longer decreases when $N_{B N}$ grows beyond 3. Instead, it remains at the same level as that of the molecule transmission. This is even more clearly demonstrated in Supplementary Figure S4 of the Supplementary Material where the cases with 5, 6, 7 and 8 layers of h-BN inside nanopores are plotted.
According to the discussion in previous section, the background transmission should attenuate in an exponential manner with increasing number of h-BN dielectric. However, our calculation now suggests that there should be some hidden interference effect that helps preserve the background transmission against the increasing thickness of h-BN dielectric.

\section{DISCUSSION}

In order to illuminate the physical mechanism for the above molecule-conducting behavior, we perform the analysis within the Landauer-NEGF formalism. Basically, there are four conducting paths from the bottom graphene to the top one given a target molecule in the nanopore:

$$
\begin{aligned}
& T_{M}=\operatorname{Tr}\left(\Gamma_{t} G_{M} \Gamma_{b} G_{M}^{+}\right) \\
& T_{h-B N}=\operatorname{Tr}\left(\Gamma_{t} G_{1, n} \Gamma_{b} G_{n, 1}^{+}\right) \\
& T_{1 M}=\operatorname{Tr}\left(\Gamma_{t} G_{1, M} \Gamma_{b} G_{M, 1}^{+}\right) \\
& T_{M n}=\operatorname{Tr}\left(\Gamma_{t} G_{M, n} \Gamma_{b} G_{n, M}^{+}\right)
\end{aligned}
$$

In the above expression, $G=\left(E-H_{\text {ext }}-\Sigma_{t}-\Sigma_{b}\right)^{-1}$ is the Green's function of the extended molecule where $\Sigma_{t}=\left[V_{t, B N}{ }^{*} ; V_{t, M^{*}}\right]$ $g_{t}\left[V_{t, B N} V_{t, M}\right]$ is the self-energy of top graphene electrode, and so is $\Sigma_{b}$ for the bottom one. $\Gamma_{t(b)}=\mathrm{i}\left(\sum_{t(b)}-\Sigma_{t(b)}{ }^{+}\right)$is the line-width function. The subscripts ${ }_{M, 1}$ and ${ }_{n}$ denote the wavefunctions of target molecule, the uppermost and lowermost h-BN layers. Thus, $G_{1, n}=$ $\left\langle\Psi_{1}\left|G^{r}\right| \Psi_{n}\right\rangle$ characterizes the propagation of electronic wavefunction from lowermost h-BN sheet $n$ to the uppermost h-BN 1, while $G_{M}=\left\langle\Psi_{M}\left|G^{r}\right| \Psi_{M}\right\rangle$ denotes the propagation in the target molecule.

Equation 23 characterizes the first conducting route where electrons/holes from the bottom graphene electrode pass through the molecule and arrive at the top one ' $b \rightarrow M \rightarrow t$ '; equation 24 denotes the second route where those carriers pass first through the lowermost sheet $n$ and then through the uppermost h-BN sheet 1 , and reach the
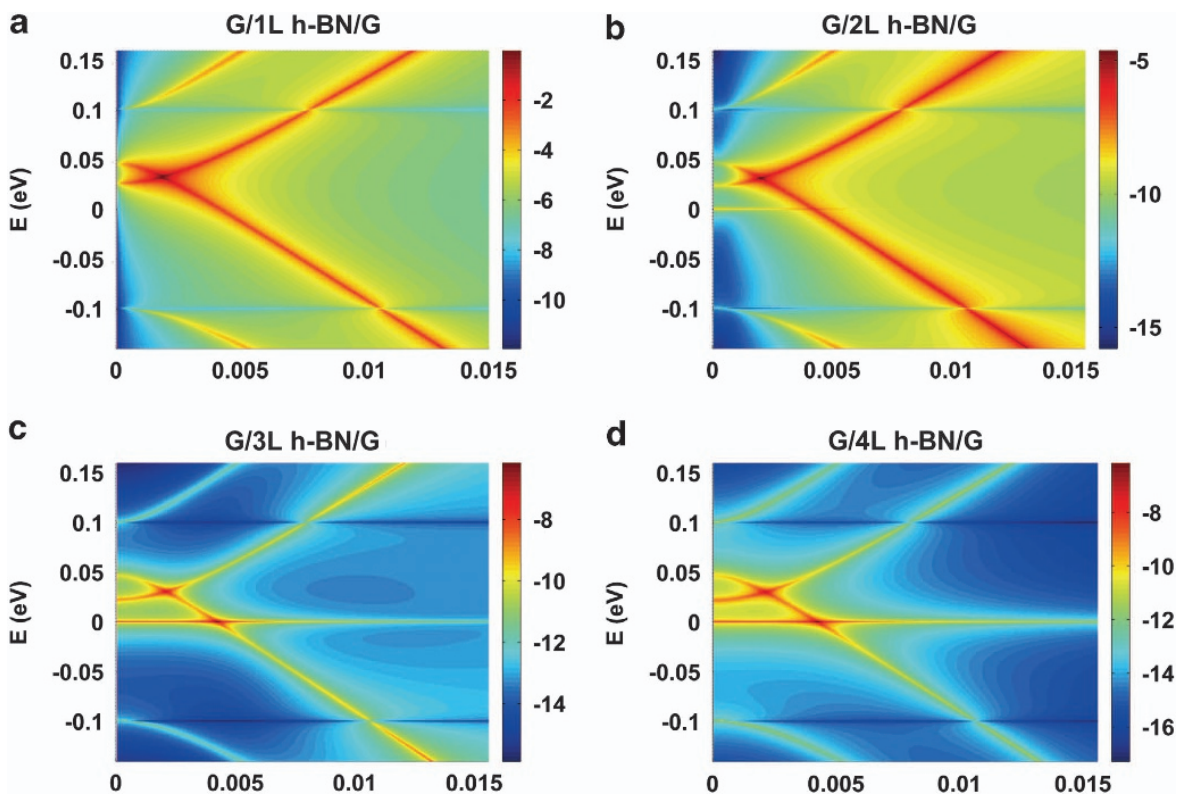

Figure 4 The molecule transmission spectrum $\log _{10} T$ when using an ABC-stacked nanopore of (a) G/1L h-BN/G; (b) G/2L h-BN/G; (c) G/3L h-BN/G; (d) G/4L $\mathrm{h}$-BN/G. Here a single-level model is used for the molecule with $E_{\mathrm{mol}}=0 \mathrm{eV}$, the applied voltage $U=0.2 \mathrm{~V}$ and the electron-doping levels of top and bottom graphene sheets are $N_{c}=10^{10} \mathrm{~cm}^{-2} \cdot 15$ 
top graphene in the end ' $b \rightarrow n \rightarrow 1 \rightarrow t$ '; equation 25 stands for the third one ' $b \rightarrow M \rightarrow 1 \rightarrow t$ '; equation 26 refers to the fourth one ' $b \rightarrow n \rightarrow M \rightarrow t$ '. Besides the four paths there exist 12 interference terms between the paths as explicitly demonstrated in the Supplementary Material.

Since the second route reveals the background transmission $T_{h-B N}$, we plot in Figure $5 \mathrm{a}$ the associated conducting diagram, while we leave the diagrams for the other three routes in the Supplementary Material for the interested readers. Both the dashed line and real line in the figure characterize propagation of electrons from the lowermost h-BN layer $n$ to the uppermost one 1 as ' $n \rightarrow 1$ '. Yet the key difference is that the former does not pass through the molecule or graphene electrodes, namely free Green's function $g_{1 n}$, while the latter does through them as one potential route of full Green's function $G_{1 n}$ ' $n \rightarrow b \rightarrow M \rightarrow t \rightarrow 1$ '. Here we remind that actually there exist quite a few propagation paths possessed by $G_{1 n}$, such as ' $n \rightarrow M \rightarrow 1$ ', ' $n \rightarrow b \rightarrow$ $M \rightarrow 1$ ' and ' $n \rightarrow M \rightarrow t \rightarrow 1$ ' (a full description of the possible routes is presented in Supplementary Figure S10 in the Supplementary Material). We deliberately choose to demonstrate the path shown in Figure $5 \mathrm{a}$ and will show our rationale in the following discussion. The strength of the propagation $g_{1 n}$ will keep decreasing with increasing number of h-BN layers as shown in the previous section. On the other hand, we are going to show that the magnitude of one propagation path of $G_{1 n}$ shown in Figure 5a is determined by the transmission through the molecule $T_{M}$. As shown by the black real line, a typical route for $G_{1 n}$ is sketched in the figure as ' $n \rightarrow b \rightarrow M \rightarrow t \rightarrow 1$ '. It involves three stages: first, electrons/holes from the lowermost h-BN layer $n$ hop to the bottom graphene electrode ' $n \rightarrow b$ '; then, they transmit to the top graphene contact via the molecule ' $b \rightarrow M \rightarrow t$ '; finally, the carriers jump from top graphene sheet to its adjacent h-BN layer 1 ' $t \rightarrow 1$ '. We remind that the intermediate stage is also characterized as $T_{M}$, while the first and last transmission processes are quite easy due to the large-area contact between the neighboring graphene and h-BN layers. Therefore, the magnitude of background transmission $T_{h-B N}$ is actually dependent on the second progress ' $b \rightarrow M \rightarrow t$ ', which is the molecule transmission $T_{M}$. In other words, given good molecule transmission $T_{M}$ the background transmission $T_{h-B N}$ will remain at the same level no matter how many h-BN layers are inside the nanopore. In a short summary, our analysis based on Green's function theory indicates that without the target molecule the background transmission $T_{h-B N}$ through the h-BN will keep decreasing with increased number of h-BN sheets inside the nanopore, as characterized by free Green's function $g_{1 n}$, while in the presence of the molecule $T_{h-B N}$ will stay largely at the same level as the molecule transmission, as marked by full Green's function $G_{1 n}$.

Figure $5 \mathrm{~b}-\mathrm{e}$ show the calculated $T_{h-B N}(E, k)$ spectrum under various layers of h-BN dielectric. The quantitative results demonstrate a sharp contrast to those without the target molecule as shown in Figure 3 in the sense that with the target molecule dwelling in, the cross-layer transmission does not keep decreasing when $N_{h-B N}$ goes beyond 3 . The calculation matches our previous analysis based on Green's function theory. We stress that the destructive/constructive quantum interference phenomena have been reported in the molecule conductance measurement. ${ }^{30}$ Here we predict its existence in the G/xL h-BN/G nanopore system. Our results are reasonable in sense yet beyond expectation, since the ordered stacking of graphene and h-BN sheets is capable of preserving the coherence of vertically propagating electronic wavefunction and thus leads to quantum interference effect, while on the other hand such behavior is definitely out of the scope of traditional role of dielectrics.

The above results suggest that $\mathrm{ABC}$-stacked $\mathrm{G} / \mathrm{h}-\mathrm{BN} / \mathrm{G}$ layers are capable of greatly reducing the background conductance in the case of open nanopore, and meanwhile they can substantially raise the overall vertical conductance when the target molecule locates within, thus enhancing the molecule measurability. Therefore, by carefully tuning the Fermi energies $E_{f}$ of top and bottom graphene electrodes through a

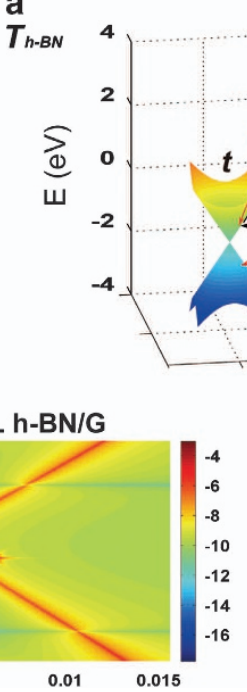

b
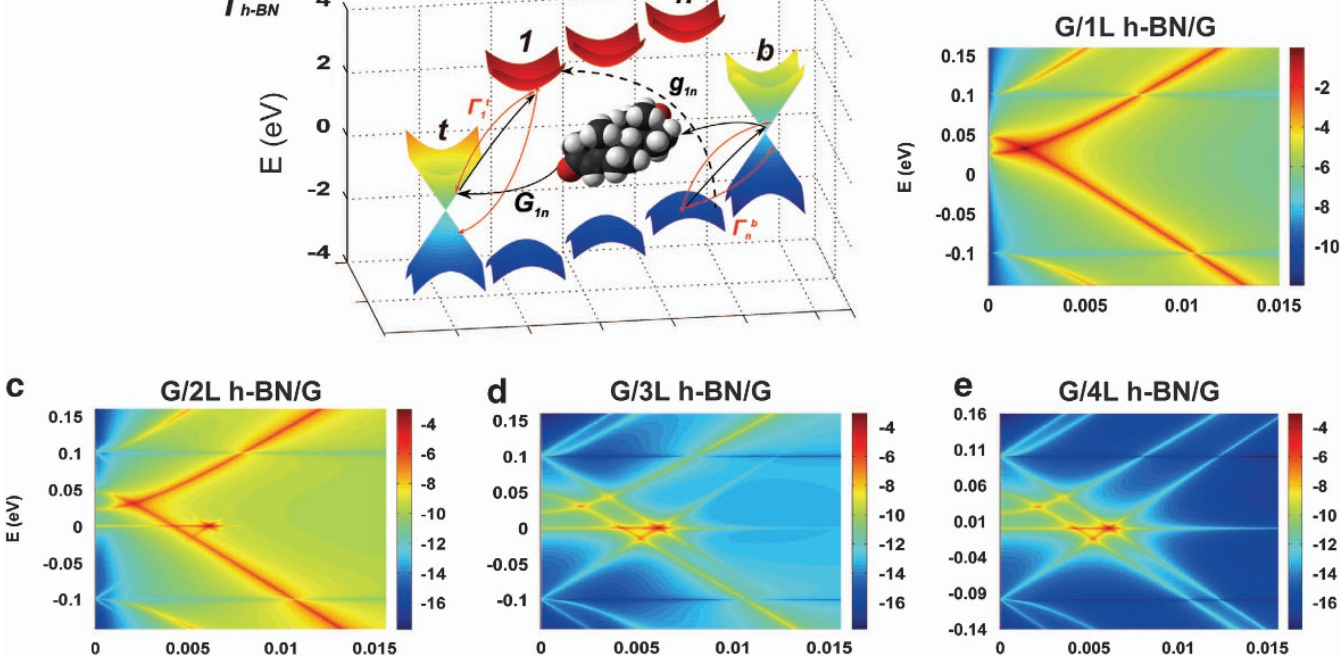

Figure 5 (a) The vertical transmission diagram through the $\mathrm{h}-\mathrm{BN}$ dielectric $T_{h-B N}$ when the target molecule dwells inside the $\mathrm{ABC}-\mathrm{stacked} \mathrm{G} / \mathrm{xL}$ h-BN/G nanopore. The superscripts ${ }^{t}$ and ${ }^{b}$ denote the top and bottom graphene sheets, while ${ }_{1}$ and $n$ mark the uppermost and lowermost $\mathrm{h}_{-\mathrm{BN}}$ layers. The red lines $\Gamma_{1}^{t}$ denote the coupling between top graphene and the uppermost h-BN layer, while the lines $\Gamma_{n}^{b}$ denote that between bottom graphene and the lowermost h-BN layer. The black dashed line indicates the propagation of electrons from the lowermost h-BN layer $n$ to the uppermost one without going through the molecule, as free Green's function $g_{1 n}$, while the black real lines indicate one potential route starting from the h-BN layer $n$, passing through the graphene electrodes and molecule, and finally arriving at the layer 1 , of full Green's function $G_{1 n}$. The calculated transmission spectrum log $10 T_{h-B N}(E, k)$ : (b) $\mathrm{G} / 1 \mathrm{~L}$ h-BN/G; (c) G/2L h-BN/G; (d) G/3L h-BN/G; (e) G/4L h-BN/G. Here all parameters are the same as in Figure 4. 


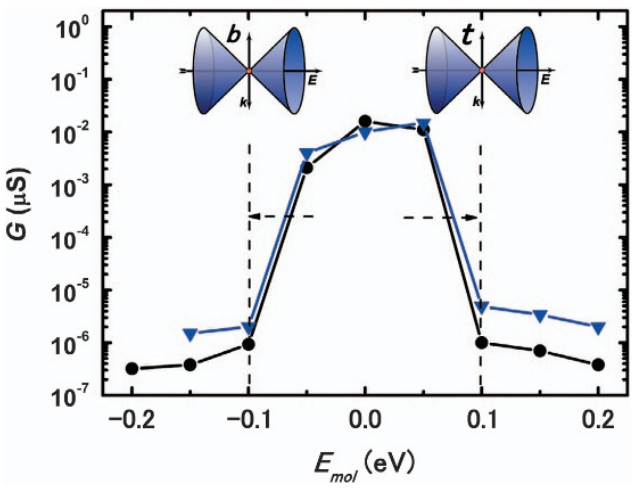

Figure 6 (a) The vertical conductance $G$ as a function of the molecule energy level $E_{m o l}$. The black line indicates G/4L h-BN/G nanopore, while the blue line indicates G/3L h-BN/G nanopore. The energy bands near $\boldsymbol{K}$ points of top $(t)$ and bottom $(b)$ graphene electrodes are also sketched.

electron/hole doping and by modulating the applied voltage $U$, the characteristic energy level of target molecules can be selected by the bias window and the h-BN dielectric will function as a signal amplifier for the single-molecule conductance. The resulting tunneling conductance is then utilized as electrical signature of the target molecule. More specifically, one of the most promising applications for nanopore sensors, namely the tunneling-conductance approach for nanopore-based DNA sequencing, calls for that those very small differences between energy levels of adenine, thymine, cytosine and guanine can be amplified on the measured conductance curves for the distinguishing purpose. ${ }^{31,32}$ Figure 6 shows our investigation on this issue where the calculated molecule conductance is plotted as a function of the molecule energy level $E_{\text {mol }}$. Here we have assumed that the coupling between the molecule and the graphene electrodes remains invariant so that the difference caused by the shift of molecule energy levels can be singled out. The effective bias window offered by the graphene electrodes is sketched in the figure. The red line shows the molecule conductance using G/3L h-BN/G nanopore and the black line for G/4L h-BN/G nanopore. It indicates that the conductance difference between molecule with $E_{\mathrm{mol}}=0 \mathrm{eV}$ and that with $E_{\mathrm{mol}}= \pm 0.1 \mathrm{eV}$ is about 4 orders. Here we remind that the bigger the difference, the better it is, as in the real experiments it is easily immersed in the aqueous environment. ${ }^{31,32}$ Thereby, our theoretical investigation demonstrates that the orderly stacked h-BN dielectric is capable of improving the molecule sensitivity of the proposed $\mathrm{G} / x \mathrm{~L}$ h-BN/G nanopore system through quantum interference effect.

In conclusion, we have proposed and shown theoretically the feasibility of $\mathrm{G} / x \mathrm{~L}$ h-BN/G nanopore sensor for single-molecule detection. The major findings are the quantum mechanical effects caused by the orderly stacked h-BN dielectric. First, the cross-layer conductance from the top graphene sheet through the h-BN layers to the bottom graphene, which is the background conductance for molecule measurement, is found to be dependent on the stacking style of the layers. The physical picture is that in the ABA stacking there is always a conducting path through the layers, while in the $\mathrm{ABC}$ case there is always no such path under small voltage and doping level. By making use of this property, we expect perfect elimination of the vertical conductance through the layers in the absence of target molecules. Second, the molecule conductance will be profoundly tuned by the sandwiched h-BN and such quantum mechanical effect is anticipated for further enhancing the sensitivity of the device.

\section{ACKNOWLEDGEMENTS}

This research is supported partially by the Japan Society for the Promotion of Science (JSPS) through its 'Funding Program for World-Leading Innovative $\mathrm{R} \& \mathrm{D}$ on Science and Technology'.

1 Postma, H. W. Rapid sequencing of individual DNA molecules in graphene nanogaps Nano Lett. 10, 420-425 (2010).

2 Garaj, S., Hubbard, W., Reina, A., Kong, J., Branton, D. \& Golovchenko, J. A. Graphene as a subnanometre trans-electrode membrane. Nature 467, 190-193 (2010).

3 Merchant, C. A., Healy, K., Wanunu, M., Ray, V., Peterman, N., Bartel, J., Fischbein, M. D., Venta, K., Luo, Z., Johnson, A. T. C. \& Drndic, M. DNA translocation through graphene nanopores. Nano Lett. 10, 2915-2921 (2010).

4 Schneider, G. F., Kowalczyk, S. W., Calado, V. E., Pandraud, G., Zandbergen, H. W., Vandersypen, L. M. K. \& Dekker, C. DNA translocation through graphene nanopores. Nano Lett. 10, 3163-3167 (2010).

5 Prasongkit, J., Grigoriev, A., Pathak, B., Ahuja, R. \& Scheicher, R. H. Transverse conductance of DNA nucleotides in a graphene nanogap from first principles. Nano Lett. 11, 1941-1945 (2011).

6 Min, S. K., Kim, W. Y., Cho, Y. \& Kim, K. S. Fast DNA sequencing with a graphene-based nanochannel device. Nat. Nanotechnol. 6, 162-165 (2011).

7 Saha, K., Drndic, M. \& Nikolic, B. DNA base-specific modulation of microampere transverse edge currents through a metallic graphene nanoribbon with a nanopore. Nano Lett. 12, 50-55 (2012).

8 Avdoshenko, S. M., Nozaki, D., da Rocha, C. G., González, J. W., Lee, M. H., Gutierrez, R. \& Cuniberti, G. Dynamic and electronic transport properties of DNA translocation through graphene nanopores. Nano Lett. 13, 1969-1976 (2013).

9 Venkatesan, B. M., Estrada, D., Banerjee, S., Jin, X., Dorgan, V. E., Bae, M.-H., Aluru, N. R., Pop, E. \& Bashir, R. Stacked graphene-Al203 nanopore sensors for sensitive detection of DNA and DNA-Protein complexes. ACS Nano 6, 441-450 (2012).

$10 \mathrm{He}$, Y., Scheicher, R. H., Grigoriev, A., Ahuja, R., Long, S., Huo, Z. \& Liu, M. Enhanced DNA sequencing performance through edge-hydrogenation of graphene electrodes. Adv. Funct. Mater. 21, 2674-2679 (2011).

11 Liu, Z., Song, L., Zhao, S., Huang, J., Ma, L., Zhang, J., Lou, J. \& Ajayan, P. M. Direct growth of graphene/hexagonal boron nitride stacked layers. Nano Lett. 11, 2032-2037 (2011).

12 Yankowitz, M., Xue, J., Cormode, D., Sanchez-Yamagishi, J. D., Watanabe, K. Taniguchi, T., Jarillo-Herrero, P., Jacquod, P. \& LeRoy, B. J. Emergence of superlattice dirac points in graphene on hexagonal boron nitride. Nat. Phys. $\mathbf{8}$, 382-386 (2012)

13 Kim, S. M., Hsu, A., Araujo, P. T., Lee, Y.-H., Palacios, T., Dresselhaus, M. Idrobo, J.-C., Kim, K. K. \& Kong, J. Synthesis of patched or stacked graphene and hBN flakes: a route to hybrid structure discovery. Nano Lett. 13, 933-941 (2013)

14 Britnell, L., Gorbachev, R. V., Jalil, R., Belle, B. D., Schedin, F., Mishchenko, A. Georgiou, T., Katsnelson, M. I., Eaves, L., Morozov, S. V., Peres, N. M. R., Leist, J. Geim, A. K., Novoselov, K. S. \& Ponomarenko, L. A. Field-effect tunneling transistor based on vertical graphene heterostructures. Science 335, 947-950 (2012).

15 Britnell, L., Gorbachev, R. V., Jalil, R., Belle, B. D., Schedin, F., Katsnelson, M. I., Eaves, L., Morozov, S. V., Mayorov, A. S., Peres, N. M. R., Castro, N., Antonio, H. Leist, J., Geim, A. K., Ponomarenko, L. A. \& Novoselov, K. S. Electron tunneling through ultrathin boron nitride crystalline barriers. Nano Lett. 12, 1707-1710 (2012)

16 Chae, D.-H., Zhang, D., Huang, X. \& von Klitzing, K. Electronic transport in two stacked graphene monolayers. Nano Lett. 12, 3905-3908 (2012).

17 Tsutsui, M., Taniguchi, M., Yokota, K. \& Kawai, T. Identifying single nucleotides by tunnelling current. Nat. Nanotechnol. 5, 286-290 (2010).

18 Huang, S., He, J., Chang, S., Zhang, P., Liang, F., Li, S., Tuchband, M., Fuhrmann, A., Ros, R. \& Lindsay, S. Identifying single bases in a DNA oligomer with electron tunnelling. Nat. Nanotechnol. 5, 868-873 (2010).

19 Zhong, X., Amorim, R. G., Scheicher, R. H., Pandey, R. \& Karna, S. P. Electronic structure and quantum transport properties of trilayers formed from graphene and boron nitride. Nanoscale 4, 5490-5498 (2012).

20 Quhe, R., Zheng, J., Luo, G., Liu, Q., Qin, R., Zhou, J., Yu, D., Nagase, S., Mei, W.-N., Gao, Z. \& Lu, J. Tunable and sizable band gap of single-layer graphene sandwiched between hexagonal boron nitride. NPG Asia Mater 4, e6 (2012).

21 Giovannetti, G., Khomyakov, P. A., Brocks, G., Kelly, P. J. \& van den Brink, J. Substrate-induced band gap in graphene on hexagonal boron nitride: ab initio density functional calculations. Phys. Rev. B 76, 073103 (2007).

22 Sakai, Y., Koretsune, T. \& Saito, S. Electronic structure and stability of layered superlattice composed of graphene and boron nitride monolayer. Phys. Rev. B 83, 205434 (2011)

23 Sławińska, J., Zasada, I. \& Klusek, Z. Energy gap tuning in graphene on hexagonal boron nitride bilayer system. Phys. Rev. B 81, 155433 (2010).

24 Stauber, T., Peres, N. M. R. \& Castro Neto, A. H. Conductivity of suspended and non-suspended graphene at finite gate voltage. Phys. Rev. B 78, 085418 (2008).

25 de Juan, F., Hwang, E. H. \& Vozmediano, M. A. H. Spectral and optical properties of doped graphene with charged impurities in the self-consistent born approximation. Phys. Rev. B 82, 245418 (2010). 
26 Park, C.-H., Giustino, F., Cohen, M. L. \& Louie, S. G. Velocity renormalization and carrier lifetime in graphene from the electron-phonon interaction. Phys. Rev. Lett. 99, 086804 (2007)

27 LeBlanc, J. P. F., Carbotte, J. P. \& Nicol, E. J. Effect of electron-phonon coupling on energy and density of states renormalizations of dynamically screened graphene. Phys. Rev. B 84, 165448 (2011)

28 Lherbier, A., Blase, X., Niquet, Y.-M., Triozon, F. \& Roche, S. Charge transport in chemically doped 2D graphene. Phys. Rev. Lett. 101, 036808 (2008).

29 Yang, W., Chen, G., Shi, Z., Liu, C., Zhang, L., Xie, G., Cheng, M., Wang, D., Yang, R., Shi, D., Watanabe, K., Taniguchi, T., Yao, Y., Zhang, Y. \& Zhang, G. Epitaxial growth of single-domain graphene on hexagonal boron nitride. Nat. Mater. 12, 792-797 (2013).

30 Guedon, C. M., Valkenier, H., Markussen, T., Thygesen, K. S., Hummelen, J. C. \& van der Molen, S. J. Observation of quantum interference in molecular charge transport. Nat. Nanotechnol. 7, 305-309 (2012).

31 Branton, D., Deamer, D. W., Marziali, A., Bayley, H., Benner, S. A., Butler, T., Di Ventra, M., Garaj, S., Hibbs, A., Huang, X., Jovanovich, S. B., Krstic, P. S., Lindsay, S., Ling, X. S., Mastrangelo, C. H., Meller, A., Oliver, J. S., Pershin, Y. V., Ramsey, J. M., Riehn, R.,
Soni, G. V., Tabard-Cossa, V., Wanunu, M., Wiggin, M. \& Schloss, J. A. The potential and challenges of nanopore sequencing. Nat. Biotechnol. 26, 1146-1153 (2008).

32 Zwolak, M. \& Di Ventra, M. Colloquium: physical approaches to DNA sequencing and detection. Rev. Mod. Phys. 80, 141-165 (2008).

(c) (i) (2) (2) This work is licensed under a Creative Commons Attribution-NonCommercial-ShareAlike 3.0 Unported License. The images or other third party material in this article are included in the article's Creative Commons license, unless indicated otherwise in the credit line; if the material is not included under the Creative Commons license, users will need to obtain permission from the license holder to reproduce the material. To view a copy of this license, visit http://creativecommons.org/licenses/by-nc-sa/3.0/

Supplementary Information accompanies the paper on the NPG Asia Materials website (http://www.nature.com/am) 